Lund-Mph-97/14

November 1997

\title{
Natural Neutrino Mass Matrix
}

\author{
Masahisa MATSUDA $\mathrm{t}^{a}$ and Morimitsu TANIMOTO $\mathrm{p}$ \\ ${ }^{a}$ Department of Mathematical Physics, LTH, Lund University, S-22100, Lund, Sweden \\ ${ }^{b}$ Science Education Laboratory, Ehime University, 790-7r Matsuyama, JAPAN
}

\begin{abstract}
Naturalness of the neutrino mass hierarchy and mixing is studied. First we select among 12 neutrino mixing patterns a few patterns, which could form the natural neutrino mass matrix. Further we show that if the Dirac neutrino mass matrix is taken as the natural one in the quark sector, then only two mixing patterns without the large mixing lead to the natural right-handed Majorana mass matrix. The rest of the chosen patterns with three degenerate mass solution lead to the unnatural right-handed Majorana mass matrix in the see-saw mechanism. Notice however, that for the chosen two natural patterns there could be a huge mass hierarchy such as $\mathcal{O}\left(10^{4 \sim 6}\right)$ in order to reproduce the inverse mass hierarchy of the light neutrinos.
\end{abstract}

\footnotetext{
${ }^{1}$ Permanent address:Department of Physics and Astronomy, Aichi University of Education, Kariya, Aichi 448, Japan. E-mail address:mmatsuda@matfys.lth.se

${ }^{2}$ E-mail address: tanimoto@edserv.ed.ehime-u.ac.jp
} 


\section{Introduction}

The standard model (SM) being extensively treated still has many unexplained features. It contains 18 parameters, among which 13 parameters belong to the flavor sector of the fermion masses and mixing [1]. It is a remarkable property of the quark and the lepton mass spectra that the masses of successive particles increase by large factors. Mixings of the quark sector seem also to have an hierarchical structure. Those features may provide an important basis for a new physics beyond the SM.

It is known that there are 54 parameters in the quark-lepton mass matrices: $M_{U}, M_{D}$ and $M_{E}$. Since only 13 combinations of 54 parameters are observable, the flavor sector is a highly undetermined system. In order to understand the pattern of fermion masses, it is necessary to reduce the number of arbitrary parameters in the Yukawa matrices from 54 to a number which is less than 13. Therefore, one asks for an organizing principle for the mass matrices. The idea of natural mass matrix, elaborated by Peccei and Wang [2], severely restricts the arbitrariness in the construction of the quark mass matrices. Here we study the natural mass matrix in the lepton sector using essentially the recent neutrino oscillation experiments [3] $\sim 14$.

Neutrino flavor oscillations are important phenomena for the study of the neutrino masses and mixing. The only possible evidences for neutrino oscillations originate from the natural beams: the solar neutrinos [3] and the atmospheric neutrinos [4] [7]. Data from the accelerator and the reactor neutrino experiments will be available in the near future. In fact data given by the LSND experiment [8] indicate at least one large mass difference. More precisely, one can expect that $\Delta m^{2} \sim \mathcal{O}\left(1 \mathrm{eV}^{2}\right)$ with $\sin ^{2} 2 \theta_{\mathrm{LSND}} \simeq 10^{-3}$. We

expect data from the KARMEN experiment [9] where the oscillations $\nu_{\mu} \rightarrow \nu_{e}\left(\bar{\nu}_{\mu} \rightarrow \bar{\nu}_{e}\right)$ are being considered as in LSND. Also we expect data from the CHORUS and NOMAD experiments [10] on the $\nu_{\mu} \rightarrow \nu_{\tau}$ oscillation. The most powerful reactor experiments 
searching for the neutrino oscillation are those of Bugey [11] and Krasnoyarsk [12] at present. These results show the region in the parameter space $\left(\sin ^{2} 2 \theta, \Delta m^{2}\right)$ where the neutrino oscillation is not observed. The first long baseline reactor experiment $\mathrm{CHOOZ}$ [13, 14] also gives us severe constraints. The possibilities of the accelerator long baseline (LBL) experiments have been discussed for example, in [15, 16]. The first experiment will begin in KEK-SuperKamiokande (K2K) [16]. Presently, the constraints are also given by the disappearance experiments in CDHS [17] and CCFR [18], and the appearance experiments E531 [19], E776 [20] and new CCFR [21]. The bounds for the neutrino masses and mixing are given in [22] [26] on the basis of the listed experiments.

Still, the neutrino masses and mixing are not determined uniquely. There should be only two hierarchical mass difference scales $\Delta m^{2}$ in the three-flavor mixing scheme. If the highest neutrino mass scale is chosen to be the LSND scale $\mathcal{O}(1 \mathrm{eV})$, the other mass scale should be either the atmospheric neutrino mass scale $\Delta m^{2} \simeq 10^{-2} \mathrm{eV}^{2}$ [4] [7] or one of the solar neutrino mass scale: $\Delta m^{2} \simeq 10^{-5} \sim 10^{-6} \mathrm{eV}^{2}$ (MSW solution) or $10^{-10} \sim 10^{-11} \mathrm{eV}^{2}$ (just-so solution) [3]. In this case, the expected neutrino masses and mixing depend on the experimental data. Since the neutrino masses and mixing angles are determined independently in those experiments, the principle of the naturalness can help us to select a few patterns of the masses and mixings among many patterns which are available from the present experimental data. Thus, we can suggest the experimental data which conduce to the natural neutrino mass matrix.

Our paper is organized as follows. In section 2 the concepts of the natural mass matrix are discussed following the arguments provided in Ref. [2] for small angle mixing case. Further we generalize these concepts to the large angle mixing case. In section 3 we study possible patterns of the neutrino masses and mixing suggested by the recent neutrino experiments. In section 4 the natural left-handed Majorana mass matrices are investigated using the patterns obtained in section 3. In section 5 a natural see-saw 
realization is considered based on the right-handed Majorana neutrinos. Finally, section 6 is devoted to the conclusions.

\section{Naturalness of the mass matrix}

The idea of the natural mass matrix was proposed in order to restrict severely the arbitrariness in the construction of the quark mass matrices [2]. This idea was worked out in the context of SUSY GUT's, when some potentially successful quark mass matrices were found at the GUT scale.

Let us consider the $2 \times 2$ Hermitian quark mass matrix $M_{i}(i=u, d)$. Assume for simplicity that it can be diagonalized by some orthogonal matrices $O_{i}(i=u, d)$ as follows:

$$
O_{i}^{T} M_{i} O_{i}=M_{i}^{\text {diag }} \equiv\left(\begin{array}{cc}
m_{i 1} & 0 \\
0 & m_{i 2}
\end{array}\right), \quad(i=u, d)
$$

with

$$
O_{i}=\left(\begin{array}{cc}
\cos \theta_{i} & \sin \theta_{i} \\
-\sin \theta_{i} & \cos \theta_{i}
\end{array}\right), \quad V_{q}=O_{u}^{T} O_{d}=\left(\begin{array}{cc}
\cos \theta_{c} & \sin \theta_{c} \\
-\sin \theta_{c} & \cos \theta_{c}
\end{array}\right)
$$

where $\theta_{c}=\theta_{d}-\theta_{u}$. Then the mass matrices can be written as

$$
M_{i}=\left(\begin{array}{cc}
c_{i}^{2} m_{i 1}+s_{i}^{2} m_{i 2} & c_{i} s_{i}\left(m_{i 2}-m_{i 1}\right) \\
c_{i} s_{i}\left(m_{i 2}-m_{i 1}\right) & s_{i}^{2} m_{i 1}+c_{i}^{2} m_{i 2}
\end{array}\right), \quad(i=u, d),
$$

where $c_{i} \equiv \cos \theta_{i}$ and $s_{i} \equiv \sin \theta_{i}$. The mixing matrix $V_{q}$ is invariant under the changes

$$
O_{d} \rightarrow O O_{d}, \quad O_{u} \rightarrow O O_{u}
$$

where $O$ is some arbitrary orthogonal matrix. Thus, using the fact that $\sin \theta_{c} \ll 1$, we can assume both $\theta_{d} \ll 1$ and $\theta_{u} \ll 1$ without loss of generality. Taking into account the quark mass hierarchy, we set

$$
m_{u}=a \lambda^{4} m_{c}, \quad m_{d}=b \lambda^{2} m_{s}
$$

where $a$ and $b$ are $\mathcal{O}(1)$ coefficients, and $\lambda \equiv \sin \theta_{c} \simeq 0.22$. Thus the mass matrices are expressed in terms of $\theta_{u}$ and $\theta_{d}$ as following:

$$
M_{u} \simeq\left(\begin{array}{cc}
a \lambda^{4}+\sin ^{2} \theta_{u} & \sin \theta_{u} \\
\sin \theta_{u} & 1
\end{array}\right) m_{c}, \quad M_{d} \simeq\left(\begin{array}{cc}
b \lambda^{2}+\sin ^{2} \theta_{d} & \sin \theta_{d} \\
\sin \theta_{d} & 1
\end{array}\right) m_{s} .
$$


There are three different options now for the angles $\theta_{u}$ and $\theta_{d}$ :

1. $\sin \theta_{d} \sim \lambda, \sin \theta_{u} \sim \lambda, \quad 2 . \sin \theta_{d} \sim \lambda, \sin \theta_{u} \leq \lambda^{2}, \quad 3 . \sin \theta_{d} \leq \lambda^{2}, \sin \theta_{u} \sim \lambda$.

The options 1 and 3 are unnatural. Indeed, one would require a severe fine-tuning of the matrix element $\left[M_{u}\right]_{11}$ forcing $\left[M_{u}\right]_{11} \simeq\left(\left[M_{u}\right]_{12}\right)^{2}$ to arrive at the large $m_{u} / m_{c} \sim \lambda^{4}$ hierarchy. On the other hand, option 2 gives a natural quark mass matrix without finetuning. Here the fine-tuning means a tuning of $\mathcal{O}\left(\lambda^{2}\right)$, which comes from the following inspection. There is a well known phenomenological relation between the CKM matrix element and the quark mass ratio as

$$
\left|V_{u s}\right| \simeq \sqrt{\frac{m_{d}}{m_{s}}} \simeq \lambda .
$$

The down quark mass ratio dominates $V_{u s}$, while the contribution of the up quark mass ratio is at most $\sqrt{m_{u} / m_{c}} \simeq \mathcal{O}\left(\lambda^{2}\right)$. This relation is consistent with the option 2. Thus, the criterion of $\mathcal{O}\left(\lambda^{2}\right)$ tuning is very useful in order to select the option 2 by excluding options 1 and 3. The success of Wolfenstein parameterization of the CKM matrix [27] guarantees that this criterion is also available for the three generation case. The extension of the natural mass matrices to the three family case in quark sector is also given in Ref. [2].

Naturalness is also expanded to the lepton sector. If neutrino sector has the hierarchical mass structure, the naturalness argument is exactly the same as in the example just described for the quark sector. However, in the neutrino sector the inverse mass hierarchy for the flavor is still allowed by the constraints obtained in the disappearance experiments of the neutrino oscillations in Bugey [11], Krasnoyarsk [12], CDHS [17] and CCFR [18]. Since the neutrino mixing is chosen to be $\sin \theta_{\nu} \sim 1$ in this case, one cannot always guarantee to have both $\theta_{\nu} \ll 1$ and $\theta_{E} \ll 1$ (charged lepton mixing) by the change in eq.(田). Therefore, we should reconsider the naturalness of the quark mass matrices in the lepton sector.

In the case of $s \simeq 1(c \ll 1)$ with $m_{1} \leq m_{2}$ in eq.(3), the mass matrix is expressed 
approximately:

$$
M_{\nu} \simeq\left(\begin{array}{cc}
m_{2} & c m_{2} \\
c m_{2} & m_{1}+c^{2} m_{2}
\end{array}\right) .
$$

The natural mass matrix without the fine-tuning requires

$$
m_{1} \geq c^{2} m_{2} .
$$

For example, if we take $c \simeq a^{\prime} \lambda, m_{1} \simeq a \lambda m_{2}$, which satisfies the condition(9), then matrix

$$
M_{\nu} \simeq\left(\begin{array}{cc}
1 & a^{\prime} \lambda \\
a^{\prime} \lambda & a \lambda
\end{array}\right) m_{2}
$$

gives us the masses $m_{2}$ and $a \lambda m_{2}$, correspondingly. Thus, the naturalness condition follows from the $(2,2)$ entry in the case of the inverse mass hierarchy of the flavor, while in the quark sector this condition follows from the $(1,1)$ entry.

Therefore it is necessary to clarify the concepts of naturalness for the large mixing angle. Furthermore, the recent experiments $[4] \sim[7]$ also indicate the large flavor mixing in the neutrinos. Let us consider the $2 \times 2$ Hermitian matrix $M_{\nu}$. Again, assume for simplicity that it can be diagonalized by some orthogonal matrix $O_{\nu}$ :

$$
O_{\nu}^{T} M_{\nu} O_{\nu}=M_{\nu}^{\text {diag }} \equiv\left(\begin{array}{cc}
m_{1} & 0 \\
0 & m_{2}
\end{array}\right) .
$$

Here the orthogonal matrix $O_{\nu}$ is

$$
O_{\nu}=\left(\begin{array}{cc}
c & s \\
-s & c
\end{array}\right), \quad c \equiv \cos \theta_{\nu} \simeq \frac{1}{\sqrt{2}}, \quad s \equiv \sin \theta_{\nu} \simeq \frac{1}{\sqrt{2}} .
$$

Then $M_{\nu}$ is expressed in terms of the mass eigenvalues and mixing angle as follows:

$$
M_{\nu}=O_{\nu} M_{\nu}^{\text {diag }} O_{\nu}^{T}=\left(\begin{array}{cc}
m_{1} c^{2}+m_{2} s^{2} & c s\left(m_{2}-m_{1}\right) \\
c s\left(m_{2}-m_{1}\right) & m_{1} s^{2}+m_{2} c^{2}
\end{array}\right) .
$$

If the neutrino masses have the hierarchy such as $m_{1} / m_{2}=\epsilon \ll 1$ with the large mixing angle, the mass matrix $M_{\nu}$ is

$$
M_{\nu} \simeq\left(\begin{array}{cc}
\epsilon c^{2}+s^{2} & c s \\
c s & c^{2}+\epsilon s^{2}
\end{array}\right) m_{2} .
$$


In the (1,1) entry, $\epsilon c^{2}$ should be fine tuned against $s^{2}$ in order to arrive at $m_{1} / m_{2} \sim \epsilon$. For example, taking $c \simeq s \simeq 1 / \sqrt{2}$, the matrix

$$
M_{\nu} \simeq\left(\begin{array}{cc}
\frac{1}{2} & \frac{1}{2} \\
\frac{1}{2} & \frac{1}{2}
\end{array}\right) m_{2}
$$

gives $m_{1} \simeq 0$ and $m_{2}$. Further if we assume that $\epsilon \simeq a \lambda^{2}$, the matrix $M_{\nu}$ in (15) should be replaced by the following matrix

$$
M_{\nu} \simeq\left(\begin{array}{cc}
\frac{1+a \lambda^{2}}{2} & \frac{1}{2} \\
\frac{1}{2} & \frac{1+a \lambda^{2}}{2}
\end{array}\right) m_{2} .
$$

The latter gives the mass $m_{1}=O\left(\lambda^{2}\right) m_{2}$ provided that the order of $O\left(\lambda^{2}\right)$ is fine-tuned. Thus, the hierarchical neutrino masses are unnatural in the case of the large mixing angle.

In the case when neutrino masses are approximately degenerate such as $m_{1} \simeq m_{2}$ and $\left(m_{2}-m_{1}\right) / m_{2}=\epsilon \ll 1$, the mass matrix $M_{\nu}$ is

$$
M_{\nu} \simeq\left(\begin{array}{cc}
1 & c s \epsilon \\
c s \epsilon & 1
\end{array}\right) m_{2} .
$$

Hence, no fine tuning is required for any entry. We can explore the same argument when the mass eigenvalues are $-m_{1}$ and $m_{2}$, in which case

$$
M_{\nu} \simeq\left(\begin{array}{cc}
c^{2} \epsilon & 2 c s \\
2 c s & s^{2} \epsilon
\end{array}\right) m_{2} .
$$

Therefore in the case of the large mixing angle we call eqs.(17,18) the natural mass matrix.

In the sequel the naturalness of the mass matrix for the lepton sector is investigated for the three family model.

\section{Pattern of neutrino masses and mixing}

The present evidences in favor of neutrino masses and mixing from solar, atmospheric and accelerator experiments cannot be all reconciled in the three family framework, unless some data are excluded. Therefore, one should classify the possible patterns of neutrino masses and mixing [28]. 
The deficit of $\nu_{e}$ source is measured in the solar neutrino experiment. The LSND experiments provide possible $\nu_{e}$ appearance by using $\nu_{\mu}$ source. The atmospheric neutrino experiment suggests the deficit of $\nu_{\mu}$ source. Thus in the framework of three family, the LSND results give us the information on $\nu_{\mu} \rightarrow \nu_{e}$ oscillations, while the atmospheric neutrino experiment provides data on $\nu_{\mu} \rightarrow \nu_{e}$ or $\nu_{\mu} \rightarrow \nu_{\tau}$ oscillations. Also $P\left(\nu_{e} \rightarrow \nu_{e}\right)$ is measured in the solar neutrino experiments.

We have the following relations for the solar neutrino [3] and the atmospheric neutrino [4] [7] and LSND [8], correspondingly:

$$
\begin{aligned}
\Delta m_{\odot}^{2} \simeq \quad(0.3 \sim 1.2) \times 10^{-5} \mathrm{eV}^{2} \\
\text { with } \quad \sin ^{2} 2 \theta_{\odot} \simeq(0.65 \sim 0.85)(\text { MSW large angle solution }) \\
\left.\quad \sin ^{2} 2 \theta_{\odot} \simeq(0.1 \sim 2) \times 10^{-2} \text { (MSW small angle solution }\right) \\
\text { or } \quad \\
\simeq \quad(5 \sim 8) \times 10^{-11} \mathrm{eV}^{2} \\
\text { with } \quad \sin ^{2} 2 \theta_{\odot} \simeq 0.8 \sim 1.0(\text { Just }- \text { so solution }) \\
\Delta m_{\text {atm }}^{2} \simeq \quad(0.3 \sim 3) \times 10^{-2} \mathrm{eV}^{2} \quad \text { with } \quad \sin ^{2} 2 \theta_{\text {atm }} \simeq(0.6 \sim 1.0), \\
\Delta m_{\mathrm{LSND}}^{2} \quad \simeq \quad 0.3 \sim 2 \mathrm{eV}^{2} \quad \text { with } \quad \sin ^{2} 2 \theta_{L S N D} \simeq(0.2 \sim 3) \times 10^{-2} .
\end{aligned}
$$

In the case of three neutrinos there is an obvious relation:

$$
\Delta m_{21}^{2}+\Delta m_{32}^{2}=\Delta m_{31}^{2},
$$

which however, is not satisfied in the view of eq.(19) unless we disregard at least one of the three above mentioned experimental results. Therefore it is impossible to reconcile all the neutrino anomalies with three neutrinos. Next we discuss the following possibilities within the three family model for light neutrinos:

(a) Choose two following solutions 


$$
\begin{aligned}
& \text { (a-1) } \Delta m_{\mathrm{LSND}}^{2}=\Delta m_{\mathrm{atm}}^{2} \sim(0.2-0.3) \mathrm{eV}^{2} \text { 29 } \\
& (\mathbf{a - 2}) \Delta m_{\odot}^{2}=\Delta m_{\mathrm{atm}}^{2} \sim 10^{-2} \mathrm{eV}^{2} \text { 30 }
\end{aligned}
$$

by stretching the data.

(b) Neglect one of the following three neutrino anomalies,

(b-1) solar neutrino data,

(b-2) LSND experimental data,

(b-3) atmospheric neutrino data.

\subsection{Case (a)}

The case(a-1) has been discussed by Cardall and Fuller [29]. The authors presented the masses and mixings:

$$
\begin{aligned}
& \Delta m_{21}^{2} \simeq 7 \times 10^{-6} \mathrm{eV}^{2}, \Delta m_{31}^{2} \simeq \Delta m_{32}^{2} \simeq 0.3 \mathrm{eV}^{2} \\
& V_{1} \simeq\left(\begin{array}{ccc}
0.994 & 0.044 & 0.100 \\
-0.108 & 0.530 & 0.841 \\
-0.015 & -0.847 & 0.532
\end{array}\right)
\end{aligned}
$$

where the MSW small angle solution of the solar neutrino is taken. The MSW large angle solution and "just-so" solution can also be accommodated in this scheme [31. For the absolute masses one expects $m_{3} \gg m_{2} \geq m_{1}$ with $m_{3} \simeq 0.5-0.6 \mathrm{eV}$. The scenario of $m_{3} \simeq m_{2} \simeq m_{1} \simeq 1 \mathrm{eV}$ is also possible.

Solution (21) requires the large values $\mathcal{O}(1 / \sqrt{2})$ for the $(2,3)$ and $(3,2)$ entries of the mixing matrix. This scenario appears to be the most natural due to the oscillation evidences. The only drawback is the behavior of zenith angle distribution in multi-GeV atmospheric data in the Kamiokande experiment. Super-Kamiokande will be able to answer the question on the zenith angle dependence. Notice, that the preliminary analysis of Super-Kamiokande already suggested this dependence in [7]. 
In the case(a-2), the smaller neutrino mass difference is accounted for both the solar and the atmospheric neutrino problems. The larger mass difference is under consideration for the LSND experiments [30]. The parameters of the mass differences and mixing are

$$
\begin{aligned}
& \Delta m_{21}^{2} \simeq 10^{-2} \mathrm{eV}^{2}, \quad \Delta m_{31}^{2} \simeq \Delta m_{32}^{2} \simeq(1-2) \mathrm{eV}^{2}, \\
& V_{2} \simeq\left(\begin{array}{ccc}
0.700 & 0.700 & 0.140 \\
-0.714 & 0.689 & 0.124 \\
-0.010 & -0.187 & 0.982
\end{array}\right) .
\end{aligned}
$$

We obtain a solution $m_{3} \gg m_{2} \geq m_{1}$ with $m_{3} \simeq \mathcal{O}(1 \mathrm{eV})$ for the absolute masses. This solution requires the large values $\mathcal{O}(1 / \sqrt{2})$ for the $(1,2)$ and $(2,1)$ entries for the mixing matrix $V_{2}$. The atmospheric deficit of $\nu_{\mu}$ is interpreted by the oscillation $\nu_{\mu} \rightarrow \nu_{e}$. Note that the recent CHOOZ experiments [14] exclude the available region for $\nu_{\mu} \rightarrow \nu_{e}$ oscillation given by Kamiokande [4] and Super-Kamiokande [7]. Although this mixing pattern might be excluded experimentally, we discuss its naturalness in the next section.

\subsection{Case (b)}

We consider the cases where one of the three neutrino anomalies (solar, atmospheric or LSND neutrino experiments) is neglected. In the following the atmospheric deficit of $\nu_{\mu}$ is interpreted as an effect of $\nu_{\mu} \rightarrow \nu_{\tau}$ oscillation by taking into account recent $\mathrm{CHOOZ}$ results [14]. We expel some mixing patterns using this remarkable result.

(1)Neglecting solar neutrino (Atmospheric neutrino+LSND):

In this scheme two cases are available:

(i) $\Delta m_{31}^{2} \simeq \Delta m_{21}^{2} \simeq \Delta m_{\mathrm{LSND}}^{2}$ and $\Delta m_{32}^{2} \simeq \Delta m_{\mathrm{atm}}^{2}$,

(ii) $\Delta m_{32}^{2} \simeq \Delta m_{31}^{2} \simeq \Delta m_{\mathrm{LSND}}^{2}$ and $\Delta m_{21}^{2} \simeq \Delta m_{\mathrm{atm}}^{2}$.

In the case (i), equation for the transition probability

$$
P\left(\nu_{\alpha} \rightarrow \nu_{\beta}\right)=\delta_{\alpha \beta}-4 \sum_{i<j} V_{\alpha i} V_{\alpha j}^{*} V_{\beta i}^{*} V_{\beta j} \sin ^{2} \frac{\Delta m_{i j}^{2} L}{4 E}
$$

gives

$$
P_{\nu_{\mu} \rightarrow \nu_{e}}^{\mathrm{LSND}}=-4 V_{\mu 1} V_{e 3} V_{\mu 3} V_{e 1} \sin ^{2} \frac{\Delta m_{31}^{2} L}{4 E}-4 V_{\mu 1} V_{e 2} V_{\mu 2} V_{e 1} \sin ^{2} \frac{\Delta m_{21}^{2} L}{4 E}
$$




$$
=4\left|V_{\mu 1}\right|^{2}\left|V_{e 1}\right|^{2} \sin ^{2} \frac{\Delta m_{\mathrm{LSND}}^{2} L}{4 E}
$$

and

$$
4\left|V_{\mu 1}\right|^{2}\left|V_{e 1}\right|^{2} \simeq(0.2 \sim 3) \times 10^{-2},
$$

where we use the unitarity relation $\sum_{i} V_{\alpha i} V_{\beta i}^{*}=\delta_{\alpha \beta}$. The atmospheric neutrino oscillation probability

$$
P_{\nu_{\mu} \rightarrow \nu_{\tau}}^{\mathrm{ATM}}=-4 V_{\mu 2} V_{\tau 3} V_{\mu 3} V_{\tau 2} \sin ^{2} \frac{\Delta m_{32}^{2} L}{4 E}
$$

imposes $-4 V_{\mu 2} V_{\tau 3} V_{\mu 3} V_{\tau 2} \simeq O(1)$. Thus we obtain approximately the maximal mixing $\left|V_{\mu 2}\right|=\left|V_{\mu 3}\right|=\left|V_{\tau 2}\right|=\left|V_{\tau 3}\right|=1 / \sqrt{2}$. For the case (ii) similar analysis is applied.

Finally this scheme needs a large mixing in $\nu_{\mu}$ and $\nu_{\tau}$ depending on the mass hierarchy pattern (i) and (ii):

$$
\begin{gathered}
m_{3} \simeq m_{2} \simeq 1 \mathrm{eV} \gg m_{1}: \quad V_{3} \simeq\left(\begin{array}{ccc}
1 & \epsilon_{1} & \epsilon_{2} \\
\epsilon_{3} & c & s \\
\epsilon_{4} & -s & c
\end{array}\right), \\
\left(\Delta m_{31}^{2} \simeq \Delta m_{21}^{2} \simeq \Delta m_{\mathrm{LSND}}^{2}, \Delta m_{32}^{2} \simeq \Delta m_{\mathrm{atm}}^{2}\right)
\end{gathered}
$$

or

$$
\begin{gathered}
m_{3} \simeq 1 \mathrm{eV} \gg m_{2} \simeq 0.1 \mathrm{eV} \geq m_{1}: \quad V_{4} \simeq\left(\begin{array}{ccc}
\epsilon_{1} & \epsilon_{2} & 1 \\
c & s & \epsilon_{3} \\
-s & c & \epsilon_{4}
\end{array}\right), \\
\left(\Delta m_{32}^{2} \simeq \Delta m_{31}^{2} \simeq \Delta m_{\mathrm{LSND}}^{2}, \Delta m_{21}^{2} \simeq \Delta m_{\mathrm{atm}}^{2}\right)
\end{gathered}
$$

respectively, where $c \equiv \cos \theta \simeq 1 / \sqrt{2}$ and $s \equiv \sin \theta \simeq 1 / \sqrt{2}$, and $\epsilon_{3} \simeq \lambda^{2} \sim \lambda^{3}$ due to the LSND data $\sin ^{2} \theta_{L S N D} \sim 10^{-3}$. Others $\epsilon_{i}$ 's are small, but not constrained at present.

\section{(2)Neglecting LSND(Solar neutrino+Atmospheric neutrino):}

In order to interpret the solar neutrino data, the component $V_{e 3}$ should be taken much smaller than 1. However, this is not compatible with the only possible solution $\nu_{\mu} \rightarrow \nu_{e}$ given by the atmospheric neutrino experiments. This solution is also excluded by the CHOOZ experiment [14] as mentioned above. Therefore, in the atmospheric neutrino 
deficit we can take either $m_{3} \simeq 0.1 \mathrm{eV}$ or the almost degenerate three neutrino masses $m_{3} \simeq m_{2} \simeq m_{1} \simeq 1 \mathrm{eV}$. For the MSW small angle solution the mixing matrix will be

$$
\begin{gathered}
\begin{array}{c}
m_{3} \simeq 0.1 \mathrm{eV} \gg m_{2} \simeq 10^{-3} \mathrm{eV} \geq m_{1}: \\
\text { or } m_{1} \simeq m_{2} \simeq m_{3} \simeq 1 \mathrm{eV}:
\end{array} \\
\left(\Delta m_{32}^{2} \simeq \Delta m_{31}^{2} \simeq \Delta m_{\mathrm{atm}}^{2}, \Delta m_{21}^{2} \simeq \Delta m_{\odot}^{2}\right),
\end{gathered}
$$

with $\epsilon_{1} \simeq \lambda^{2}$, which is due to $\sin ^{2} 2 \theta_{\odot} \simeq 10^{-2}$. Another solution is

$$
V_{6} \simeq\left(\begin{array}{ccc}
\epsilon_{1} & 1 & \epsilon_{2} \\
c & \epsilon_{3} & s \\
-s & \epsilon_{4} & c
\end{array}\right)
$$

where either of the following three cases:

$$
\begin{aligned}
& m_{3} \simeq m_{2} \gg m_{1}\left(\Delta m_{31}^{2} \simeq \Delta m_{21}^{2} \simeq \Delta m_{\mathrm{atm}}^{2}, \Delta m_{32}^{2} \simeq \Delta m_{\odot}^{2}\right) \\
& m_{3} \gg m_{2} \geq m_{1}\left(\Delta m_{32}^{2} \simeq \Delta m_{31}^{2} \simeq \Delta m_{\mathrm{atm}}^{2}, \Delta m_{21}^{2} \simeq \Delta m_{\odot}^{2}\right) \\
& m_{1} \simeq m_{2} \simeq m_{3} \simeq 1 \mathrm{eV}
\end{aligned}
$$

is allowed.

On the other hand, a typical mixing matrix pattern in the case of the just-so solution and the MSW large angle solution has the following form:

$$
\left(\begin{array}{ccc}
c & s & \epsilon_{1} \\
-s & c & \epsilon_{2} \\
\epsilon_{3} & \epsilon_{4} & 1
\end{array}\right) \quad \text { or } \quad\left(\begin{array}{ccc}
c & s & \epsilon_{1} \\
\epsilon_{2} & \epsilon_{3} & 1 \\
-s & c & \epsilon_{4}
\end{array}\right) .
$$

However, neither of these matrices is compatible with the maximal mixing pattern of the atmospheric $\nu_{\mu} \rightarrow \nu_{\tau}$ oscillation. Of course one can find a mixing matrix which is consistent with both large angles of the solar neutrino and the atmospheric neutrino within the experimental error bars [32]. But again, this case is too complicated for the analysis of the naturalness of the mixing matrix. We shall not discuss it in the following.

Thus the MSW small angle solution in the solar neutrino and the atmospheric $\nu_{\mu} \rightarrow$ $\nu_{\tau}$ oscillation solution are compatible with the mixing matrix $V_{5,6}$. We investigate the naturalness of these two mixing patterns in section 4 . 
(3)Neglecting atmospheric neutrino(Solar neutrino $+L S N D)$ :

The standard scenario is characterized by either the strong mass hierarchy $m_{3} \gg$ $m_{2} \gg m_{1}$, or the $m_{2}-m_{1}$ degenerate case where $m_{3} \gg m_{2} \simeq m_{1}\left(\Delta m_{32}^{2} \simeq \Delta m_{31}^{2} \simeq\right.$ $\left.\Delta m_{\mathrm{LSND}}^{2}, \Delta m_{21}^{2} \simeq \Delta m_{\odot}^{2}\right)$. Then the possible mixing matrices are

$$
\begin{aligned}
V_{7} \simeq\left(\begin{array}{ccc}
1 & \epsilon_{1} & \epsilon_{2} \\
\epsilon_{3} & 1 & \epsilon_{4} \\
\epsilon_{5} & \epsilon_{6} & 1
\end{array}\right), & V_{8} \simeq\left(\begin{array}{ccc}
\epsilon_{1} & 1 & \epsilon_{2} \\
1 & \epsilon_{3} & \epsilon_{4} \\
\epsilon_{5} & \epsilon_{6} & 1
\end{array}\right), \\
V_{9} \simeq\left(\begin{array}{ccc}
\epsilon_{1} & 1 & \epsilon_{2} \\
\epsilon_{3} & \epsilon_{4} & 1 \\
1 & \epsilon_{5} & \epsilon_{6}
\end{array}\right), & V_{10} \simeq\left(\begin{array}{ccc}
1 & \epsilon_{1} & \epsilon_{2} \\
\epsilon_{3} & \epsilon_{4} & 1 \\
\epsilon_{5} & 1 & \epsilon_{6}
\end{array}\right) .
\end{aligned}
$$

for the MSW small mixing solution in the solar neutrino experiments. Here we have $\epsilon_{2} \simeq \lambda$, $\epsilon_{4} \simeq \lambda^{3 / 2}$ for $V_{7}$ and $V_{8}$ where $\epsilon_{2} \epsilon_{4}$ and $\epsilon_{4}$ are constrained from the LSND data $\left(\nu_{\mu}-\nu_{e}\right)$ and CHORUS/NOMAD data $\left(\nu_{\mu}-\nu_{\tau}\right)$, respectively. The relation $\epsilon_{2} \simeq \lambda^{2} \sim \lambda^{3}$ for $V_{9}$ and $V_{10}$ is given by the LSND data. The mixing matrices $V$ with the $(1,3)$ entry, being nearly 1 , are not allowed by the solar neutrino deficit [22] [26].

On the other hand, taking the just-so or the MSW large angle solutions, we obtain the following matrices

$$
V_{11}=\left(\begin{array}{ccc}
c & s & \epsilon_{1} \\
-s & c & \epsilon_{2} \\
\epsilon_{3} & \epsilon_{4} & 1
\end{array}\right) \quad \text { or } \quad V_{12}=\left(\begin{array}{ccc}
c & s & \epsilon_{1} \\
\epsilon_{2} & \epsilon_{3} & 1 \\
-s & c & \epsilon_{4}
\end{array}\right),
$$

where $\epsilon_{1} \simeq \lambda$ and $\epsilon_{2} \simeq \lambda^{3 / 2}$ for $V_{11}$ and $\epsilon_{1} \simeq \lambda^{2} \sim \lambda^{3}$ for $V_{12}$ due to the LSND data and CHORUS/NOMAD data. Notice, that matrices in (34) require small values for $V_{e 3} V_{\mu 3}$.

\section{Natural lepton mass matrix}

We discuss here the mass matrices of the charged lepton $M_{E}$ and the light Majorana neutrino $M_{L L}$, in which $C P$ violation is neglected. Let us begin with the charged lepton sector. The mass matrix $M_{E}$ is diagonalized by the orthogonal matrix $O_{E}$, which is parameterized in terms of three angles:

$$
O_{E}=\left(\begin{array}{ccc}
c_{13} c_{12} & c_{13} s_{12} & s_{13} \\
-c_{23} s_{12}-s_{23} s_{13} c_{12} & c_{23} c_{12}-s_{23} s_{13} s_{12} & s_{23} c_{13} \\
s_{23} s_{12}-c_{23} s_{13} c_{12} & -s_{23} c_{12}-c_{23} s_{13} s_{12} & c_{23} c_{13}
\end{array}\right),
$$


where $s_{i j} \equiv \sin \theta_{i j}$ and $c_{i j} \equiv \cos \theta_{i j}$ are mixings, and the $C P$ violating phase is neglected.

For the charged leptons, the mass hierarchy is clearly determined as follows:

$$
\frac{m_{e}}{m_{\mu}} \simeq 2 \times \lambda^{4}, \quad \frac{m_{\mu}}{m_{\tau}} \simeq \lambda^{2}
$$

Choosing properly an orthogonal matrix, we can satisfy the relation $\theta_{i j} \ll 1$ due to the large mass hierarchy. Next applying the same naturalness arguments as in section 2, we get $\theta_{12} \leq \lambda^{2}$, just as in the case of the up-quark sector. We derive $\theta_{23} \leq \lambda$ and $\theta_{13} \leq \lambda^{3}$ for the rest possible angles. Finally, we obtain the orthogonal matrix which diagonalizes $M_{E}$ :

$$
\left|O_{E}\right| \simeq\left(\begin{array}{ccc}
1 & \leq \lambda^{2} & \leq \lambda^{3} \\
\leq \lambda^{2} & 1 & \leq \lambda \\
\leq \lambda^{3} & \leq \lambda & 1
\end{array}\right)
$$

Using this orthogonal matrix $O_{E}$, we can study now the natural neutrino mass matrix. Recall, that there are some expected patterns of neutrino masses and mixing(see section 3). We shall investigate the naturalness of those patterns. Our strategy will be the following. At the first stage we construct the light Majorana neutrino mass matrix

$$
M_{L L}=O_{\nu}^{T} M_{L L}^{\mathrm{diag}} O_{\nu}
$$

Matrix $O_{\nu}$ is obtained from the relation $V=O_{E}^{T} O_{\nu}$. Note, that various $V$ 's were discussed in section 3. More precisely, there are the cases (a) and (b) under the assumption of the $C P$ conservation in the lepton sector. Thus, we can argue now the naturalness of $M_{L L}$. If the relevant $M_{L L}$ is a natural mass matrix at this stage, we refer to this as the first stage natural mass matrix. Then we proceed to the second stage described below in section 5 .

In the see-saw mechanism [33], one obtains $M_{L L}$ as

$$
M_{L L}=M_{L R} M_{R R}^{-1} M_{L R}^{T},
$$

where $M_{L R}$ is the Dirac mass matrix and $M_{R R}$ is the right-handed Majorana mass matrix. We argue the naturalness of the $M_{R R}$ leading to $M_{L L}$ using the hierarchical natural mass matrix $M_{L R}$, obtained by Peccei and Wang [2] for the up and the down quark sectors. If there is no fine-tuning in $M_{R R}$, we call $M_{L L}$ the second stage natural matrix. 


\subsection{Case(a)}

Consider the Cardall and Fuller mixing matrix $V_{1}$. The orthogonal matrix $O_{\nu}$ is expected to be

$$
O_{\nu} \simeq\left(\begin{array}{ccc}
1 & \epsilon_{1} & \epsilon_{2} \\
\epsilon_{3} & c & s \\
\epsilon_{4} & -s & c
\end{array}\right)
$$

where $\epsilon_{1} \sim \lambda^{2}, \epsilon_{2} \sim \lambda, \epsilon_{3} \sim-\lambda, \epsilon_{4} \sim \lambda^{2}$ and $c \equiv \cos \theta \simeq 0.5$ according to $O_{\nu}=O_{E} V$ with a fixed natural orthogonal matrix

$$
O_{E}=\left(\begin{array}{ccc}
1 & \lambda^{2} & -\lambda^{3} \\
-\lambda^{2} & 1 & \lambda^{2} \\
\lambda^{3} & -\lambda^{2} & 1
\end{array}\right)
$$

Then constructed $M_{L L}$ can be rewritten as follows:

$$
M_{L L}^{(1)} \simeq\left(\begin{array}{ccc}
m_{1}+m_{2} \epsilon_{3}^{2}+m_{3} \epsilon_{4}^{2} & m_{1} \epsilon_{1}+m_{2} c \epsilon_{3}-m_{3} s \epsilon_{4} & m_{1} \epsilon_{2}+m_{2} s \epsilon_{3}+m_{3} c \epsilon_{4} \\
m_{1} \epsilon_{1}+m_{2} c \epsilon_{3}-m_{3} s \epsilon_{4} & m_{1} \epsilon_{1}^{2}+m_{2} c^{2}+m_{3} s^{2} & m_{1} \epsilon_{1} \epsilon_{2}+c s\left(m_{2}-m_{3}\right) \\
m_{1} \epsilon_{2}+m_{2} s \epsilon_{3}+m_{3} c \epsilon_{4} & m_{1} \epsilon_{1} \epsilon_{2}+c s\left(m_{2}-m_{3}\right) & m_{1} \epsilon_{2}^{2}+m_{2} s^{2}+m_{3} c^{2}
\end{array}\right) .
$$

The naturalness condition of the $3 \times 3$ mass matrix in eq.(42) is obtained from the following inspection. The $m_{1}, m_{2}$ and $m_{3}$ terms with coefficients $\mathcal{O}(1)$ on the diagonal entries are essential ones for the mass eigenvalues $m_{1}, m_{2}$ and $m_{3}$ as we have seen in section 2 . Hence, we cannot neglect these terms. Then the naturalness of the matrix is easily found focusing on $m_{1}, m_{2}$ and $m_{3}$ terms with coefficients $\mathcal{O}(1)$ on the diagonal entries. In order to avoid the fine-tuning of $(1,1),(2,2)$ and $(3,3)$ entries in $M_{L L}$, we find the conditions

$$
m_{1} \geq m_{2} \epsilon_{3}^{2}+m_{3} \epsilon_{4}^{2}, \quad m_{2} c^{2} \geq m_{1} \epsilon_{1}^{2}+m_{3} s^{2}, \quad m_{3} c^{2} \geq m_{1} \epsilon_{2}^{2}+m_{2} s^{2}
$$

which are satisfied as long as $m_{3} \simeq m_{2}$ due to $c \simeq 0.5$. Taking into consideration the relations $\Delta m_{21}^{2} \simeq 7 \times 10^{-6} \mathrm{eV}^{2}$ and $\Delta m_{31}^{2} \simeq \Delta m_{32}^{2} \simeq 0.3 \mathrm{eV}^{2}$ in the Cardall and Fuller scheme, we obtain

$$
m_{3} \simeq m_{2} \simeq m_{1}
$$

In other words, the hierarchical neutrino masses are excluded by the principle of the naturalness of the mass matrix. The three neutrino masses should be almost degenerated. This result is still valid for a few variations of the mixing matrix [31]. 
In the case (a-2) where $\Delta m_{\odot}^{2}=\Delta m_{\text {atm }}^{2}$ is required [30], the analysis is similar to the previous case. The orthogonal matrix $O_{\nu}=O_{E} V_{2}$ is

$$
O_{\nu} \simeq\left(\begin{array}{ccc}
c & s & \epsilon_{1} \\
-s & c & \epsilon_{2} \\
\epsilon_{3} & \epsilon_{4} & 1
\end{array}\right)
$$

where $\epsilon_{1} \sim \epsilon_{2} \sim-\epsilon_{3} \sim-\epsilon_{4} \sim \lambda$ and $c \simeq s \simeq 1 / \sqrt{2}$. The $M_{L L}$ is

$$
M_{L L}^{(2)} \simeq\left(\begin{array}{ccc}
m_{1} c^{2}+m_{2} s^{2}+m_{3} \epsilon_{3}^{2} & \left(m_{1}-m_{2}\right) c s+m_{3} c \epsilon_{3} \epsilon_{4} & m_{1} c \epsilon_{1}-m_{2} s \epsilon_{2}+m_{3} \epsilon_{3} \\
\left(m_{1}-m_{2}\right) c s+m_{3} c \epsilon_{3} \epsilon_{4} & m_{1} s^{2}+m_{2} c^{2}+m_{3} \epsilon_{4}^{2} & m_{1} s \epsilon_{1}+m_{2} c \epsilon_{2}+m_{3} \epsilon_{4} \\
m_{1} c \epsilon_{1}-m_{2} s \epsilon_{2}+m_{3} \epsilon_{3} & m_{1} s \epsilon_{1}+m_{2} c \epsilon_{2}+m_{3} \epsilon_{4} & m_{1} \epsilon_{1}^{2}+m_{2} \epsilon_{2}^{2}+m_{3}
\end{array}\right),
$$

derived from eq.(38). Using this matrix, we can put the naturalness condition for $(1,1)$, $(2,2)$ and $(3,3)$ entries as

$$
m_{1} c^{2} \geq m_{2} s^{2}+m_{3} \epsilon_{3}^{2}, \quad m_{2} c^{2} \geq m_{1} s^{2}+m_{3} \epsilon_{3}^{2}, \quad m_{3} \geq m_{1} \epsilon_{1}^{2}+m_{2} \epsilon_{2}^{2},
$$

which leads to

$$
m_{3} \gg m_{2} \simeq m_{1} \quad \text { or } \quad m_{3} \simeq m_{2} \simeq m_{1}
$$

The first case of the mass patterns require the condition $m_{1} / m_{3} \simeq m_{2} / m_{3} \sim \epsilon_{3}^{2} \sim \lambda^{2}$. Thus $V_{2}$ could be a natural one. The latter case requires the neutrino masses to be much larger than $1 \mathrm{eV}$ in order to reproduce $\Delta m_{21}^{2} \simeq 10^{-2} \mathrm{eV}^{2}$ and $\Delta m_{31}^{2} \simeq \Delta m_{32}^{2} \simeq(1-2) \mathrm{eV}^{2}$. However, these masses contradict to the neutrinoless double beta decay experiments [34]. Therefore we do not consider this case in our paper.

Table 1 summarizes the results on the naturalness for $V_{1}$ and $V_{2}$.

$$
\text { Table } 1
$$

\subsection{Case(b)}

(1)Neglecting solar neutrino (Atmospheric neutrino $+L S N D$ ):

In the case of $V_{3}$ in eq.(27), the orthogonal matrix $O_{\nu}$ is expected to be

$$
O_{\nu} \simeq\left(\begin{array}{ccc}
1 & \epsilon_{1} & \epsilon_{2} \\
\epsilon_{3} & c & s \\
\epsilon_{4} & -s & c
\end{array}\right)
$$


where $\epsilon_{3} \sim \lambda^{2}$ and $c \simeq s \simeq 1 / \sqrt{2}$. The neutrino masses satisfy the hierarchy $m_{3} \simeq m_{2} \gg$ $m_{1}$. The matrix $M_{L L}^{(3)}$ is the same as $M_{L L}^{(1)}$ in eq.(42), and the conditions of naturalness are the same as in eq.(43). This is consistent with the hierarchy $m_{3} \simeq m_{2} \gg m_{1}$ assuming the condition $m_{1} \geq m_{2} \epsilon_{3}^{2}+m_{3} \epsilon_{4}^{2}$. Therefore, $V_{3}$ leads to a natural mass matrix.

In the case of $V_{4}$ in eq.(28), the orthogonal matrix $O_{\nu}$ is expected to be

$$
O_{\nu} \simeq\left(\begin{array}{ccc}
\epsilon_{1} & \epsilon_{2} & 1 \\
c & s & \epsilon_{3} \\
-s & c & \epsilon_{4}
\end{array}\right)
$$

with $m_{3} \gg m_{2} \geq m_{1}$. Then, $M_{L L}$ is given by

$$
M_{L L}^{(4)} \simeq\left(\begin{array}{ccc}
m_{1} \epsilon_{1}^{2}+m_{2} c^{2}+m_{3} s^{2} & m_{1} \epsilon_{1} \epsilon_{2}+\left(m_{2}-m_{3}\right) c s & m_{1} \epsilon_{1}+m_{2} c \epsilon_{3}-m_{3} s \epsilon_{4} \\
m_{1} \epsilon_{1} \epsilon_{2}+\left(m_{2}-m_{3}\right) c s & m_{1} \epsilon_{2}^{2}+m_{2} s^{2}+m_{3} c^{2} & m_{1} \epsilon_{2}+m_{2} s \epsilon_{3}+m_{3} c \epsilon_{4} \\
m_{1} \epsilon_{1}+m_{2} c \epsilon_{3}-m_{3} s \epsilon_{4} & m_{1} \epsilon_{2}+m_{2} s \epsilon_{3}+m_{3} c \epsilon_{4} & m_{1}+m_{2} \epsilon_{3}^{2}+m_{3} \epsilon_{4}^{2}
\end{array}\right) .
$$

We derive the conditions of naturalness:

$$
m_{2} c^{2} \geq m_{1} \epsilon_{1}^{2}+m_{3} s^{2}, \quad m_{3} c^{2} \geq m_{1} \epsilon_{2}^{2}+m_{2} s^{2}, \quad m_{1} \geq m_{2} \epsilon_{3}^{2}+m_{3} \epsilon_{4}^{2} .
$$

These require $m_{3} \simeq m_{2} \gg m_{1}$, which contradicts to $m_{3} \gg m_{2} \geq m_{1}$ determined by eq. 28). Thus, $V_{4}$ leads to an unnatural mass matrix.

\section{(2)Neglecting LSND(Solar neutrino+Atmospheric neutrino):}

In the case of $V_{5}$ in eq.(29), the orthogonal matrix $O_{\nu}$ is expected to be

$$
O_{\nu} \simeq\left(\begin{array}{ccc}
1 & \epsilon_{1} & \epsilon_{2} \\
\epsilon_{3} & c & s \\
\epsilon_{4} & -s & c
\end{array}\right)
$$

where $\epsilon_{1} \sim \lambda^{2}$ and $c \simeq s \simeq 1 / \sqrt{2}$. The neutrino masses satisfy either the hierarchy $m_{3} \gg m_{2} \geq m_{1}$ or $m_{3} \simeq m_{2} \simeq m_{1}$. The $M_{L L}^{(5)}$ is the same as $M_{L L}^{(1)}$ in eq.(42). Then the conditions of the natural mass matrix are also the same as in eq.(43). This is in consistence with the case of $m_{3} \simeq m_{2} \simeq m_{1}$. Therefore, $V_{5}$ leads to a natural mixing matrix for the degenerate masses, but does not for $m_{3} \gg m_{2} \geq m_{1}$.

In the case of $V_{6}$ in eq.(30), the orthogonal matrix $O_{\nu}$ is

$$
O_{\nu} \simeq\left(\begin{array}{ccc}
\epsilon_{1} & 1 & \epsilon_{2} \\
c & \epsilon_{3} & s \\
-s & \epsilon_{4} & c
\end{array}\right)
$$


where $\epsilon_{1} \sim \lambda^{2}$ and $c \simeq s \simeq 1 / \sqrt{2}$ with $m_{3} \simeq m_{2} \gg m_{1}$. Then $M_{L L}$ is given by

$$
M_{L L}^{(6)} \simeq\left(\begin{array}{ccc}
m_{1} \epsilon_{1}^{2}+m_{2} c^{2}+m_{3} s^{2} & m_{1} \epsilon_{1}+m_{2} c \epsilon_{3}-m_{3} s \epsilon_{4} & m_{1} \epsilon_{1} \epsilon_{2}+\left(m_{2}-m_{3}\right) c s \\
m_{1} \epsilon_{1}+m_{2} c \epsilon_{3}-m_{3} s \epsilon_{4} & m_{1}+m_{2} \epsilon_{3}^{2}+m_{3} \epsilon_{4}^{2} & m_{1} \epsilon_{2}+m_{2} s \epsilon_{3}+m_{3} c \epsilon_{4} \\
m_{1} \epsilon_{1} \epsilon_{2}+\left(m_{2}-m_{3}\right) c s & m_{1} \epsilon_{2}+m_{2} s \epsilon_{3}+m_{3} c \epsilon_{4} & m_{1} \epsilon_{2}^{2}+m_{2} s^{2}+m_{3} c^{2}
\end{array}\right) .
$$

Considering the $(2,2)$ entry of the matrix, we conclude that the natural mass matrix is defined correctly if

$$
m_{1} \geq m_{2} \epsilon_{3}^{2}+m_{3} \epsilon_{4}^{2}
$$

The latter is satisfied for the both cases: $m_{3} \simeq m_{2} \gg m_{1}$ and $m_{3} \simeq m_{2} \simeq m_{1}$. Thus $V_{6}$ leads to a natural mass matrix for both $m_{3} \simeq m_{2} \gg m_{1}$ and $m_{3} \simeq m_{2} \simeq m_{1}$.

\section{(3)Neglecting the atmospheric neutrino(Solar neutrino $+L S N D)$ :}

The standard scenario is characterized by the strong mass hierarchy $m_{3} \gg m_{2} \geq m_{1}$. For the case of $V_{7}$ in eq.(33), we obtain the constrains from the solar neutrino and the LSND data: $\epsilon_{1} \leq \lambda^{2}, \epsilon_{2} \sim \lambda, \epsilon_{4} \leq \lambda^{3 / 2}$. Then, the $M_{L L}$ matrix is

$$
M_{L L}^{(7)} \simeq\left(\begin{array}{ccc}
m_{1}+m_{2} \epsilon_{3}^{2}+m_{3} \epsilon_{5}^{2} & m_{1} \epsilon_{1}+m_{2} \epsilon_{3}+m_{3} \epsilon_{5} \epsilon_{6} & m_{1} \epsilon_{2}+m_{2} \epsilon_{3} \epsilon_{4}+m_{3} \epsilon_{5} \\
m_{1} \epsilon_{1}+m_{2} \epsilon_{3}+m_{3} \epsilon_{5} \epsilon_{6} & m_{1} \epsilon_{1}^{2}+m_{2}+m_{3} \epsilon_{6}^{2} & m_{1} \epsilon_{1} \epsilon_{2}+m_{2} \epsilon_{4}+m_{3} \epsilon_{6} \\
m_{1} \epsilon_{2}+m_{2} \epsilon_{3} \epsilon_{4}+m_{3} \epsilon_{5} & m_{1} \epsilon_{1} \epsilon_{2}+m_{2} \epsilon_{4}+m_{3} \epsilon_{6} & m_{1} \epsilon_{2}^{2}+m_{2} \epsilon_{4}^{2}+m_{3}
\end{array}\right) .
$$

In order to avoid the fine-tuning, one should impose the following conditions for the $(1,1)$ and $(2,2)$ entries:

$$
m_{1} \geq m_{2} \epsilon_{3}^{2}+m_{3} \epsilon_{5}^{2}, \quad m_{2} \geq m_{1} \epsilon_{1}^{2}+m_{3} \epsilon_{6}^{2}
$$

Choosing $\epsilon_{3} \leq \lambda^{2}, \epsilon_{5} \sim \lambda$ and $\epsilon_{6} \leq \lambda^{2}$ we satisfy these conditions in the case where $m_{1} \simeq$ $m_{2} \lambda \simeq m_{3} \lambda^{2}$. However this hierarchy is not sufficient to ensure $\Delta m_{32}^{2} \simeq \Delta m_{31}^{2} \simeq \Delta m_{L S N D}^{2}$ and $\Delta m_{21}^{2} \simeq \Delta m_{\odot}^{2}$. But the latter contradicts to the mass hierarchy $m_{3} \gg m_{2} \gg m_{1}$. Thus $V_{7}$ leads to an unnatural mass matrix.

For $V_{8}$ in eq.(33) we obtain

$$
M_{L L}^{(8)} \simeq\left(\begin{array}{ccc}
m_{1} \epsilon_{1}^{2}+m_{2}+m_{3} \epsilon_{5}^{2} & m_{1} \epsilon_{1}+m_{2} \epsilon_{3}+m_{3} \epsilon_{5} \epsilon_{6} & m_{1} \epsilon_{1} \epsilon_{2}+m_{2} \epsilon_{4}+m_{3} \epsilon_{5} \\
m_{1} \epsilon_{1}+m_{2} \epsilon_{3}+m_{3} \epsilon_{5} \epsilon_{6} & m_{1}+m_{2} \epsilon_{3}^{2}+m_{3} \epsilon_{6}^{2} & m_{1} \epsilon_{2}+m_{2} \epsilon_{3} \epsilon_{4}+m_{3} \epsilon_{6} \\
m_{1} \epsilon_{1} \epsilon_{2}+m_{2} \epsilon_{4}+m_{3} \epsilon_{5} & m_{1} \epsilon_{2}+m_{2} \epsilon_{3} \epsilon_{4}+m_{3} \epsilon_{6} & m_{1} \epsilon_{2}^{2}+m_{2} \epsilon_{4}^{2}+m_{3}
\end{array}\right)
$$


which leads to an unnatural mass matrix. Indeed, the naturalness conditions

$$
m_{2} \geq m_{1} \epsilon_{1}^{2}+m_{3} \epsilon_{5}^{2}, \quad m_{1} \geq m_{2} \epsilon_{3}^{2}+m_{3} \epsilon_{6}^{2},
$$

cannot be satisfied for the mass hierarchy $m_{3} \gg m_{2} \geq m_{1}$ according to the similar argument in the previous case. Thus, we find that $V_{8}$ is unnatural as well as $V_{7}$.

For $V_{9}$ in eq.(33), we derive

$$
M_{L L}^{(9)} \simeq\left(\begin{array}{ccc}
m_{1} \epsilon_{1}^{2}+m_{2} \epsilon_{3}^{2}+m_{3} & m_{1} \epsilon_{1}+m_{2} \epsilon_{3} \epsilon_{4}+m_{3} \epsilon_{5} & m_{1} \epsilon_{1} \epsilon_{2}+m_{2} \epsilon_{3}+m_{3} \epsilon_{6} \\
m_{1} \epsilon_{1}+m_{2} \epsilon_{3} \epsilon_{4}+m_{3} \epsilon_{5} & m_{1}+m_{2} \epsilon_{4}^{2}+m_{3} \epsilon_{5}^{2} & m_{1} \epsilon_{2}+m_{2} \epsilon_{4}+m_{3} \epsilon_{5} \epsilon_{6} \\
m_{1} \epsilon_{1} \epsilon_{2}+m_{2} \epsilon_{3}+m_{3} \epsilon_{6} & m_{1} \epsilon_{2}+m_{2} \epsilon_{4}+m_{3} \epsilon_{5} \epsilon_{6} & m_{1} \epsilon_{2}^{2}+m_{2}+m_{3} \epsilon_{6}^{2}
\end{array}\right),
$$

and the corresponding naturalness conditions

$$
m_{2} \geq m_{1} \epsilon_{2}^{2}+m_{3} \epsilon_{6}^{2}, \quad m_{1} \geq m_{2} \epsilon_{4}^{2}+m_{3} \epsilon_{5}^{2} .
$$

These conditions are similar to the cases of $V_{7,8}$, and we can choose arbitrary small values $\epsilon_{5}$ and $\epsilon_{6}$, keeping $\epsilon_{6} \geq \epsilon_{5}$. For example, as in the case $m_{3} \gg m_{2} \simeq m_{1}$, we obtain the mixing matrix

$$
V_{9} \simeq\left(\begin{array}{ccc}
-\lambda^{2} & 1 & \lambda^{3} \\
-\lambda^{2} & -\lambda^{3} & 1 \\
1 & \lambda^{2} & \lambda^{2}
\end{array}\right)
$$

which is consistent with the condition(62). Here we input $\epsilon_{2} \simeq \lambda^{3}, \epsilon_{5} \simeq \epsilon_{6} \simeq \lambda^{2}$, and the masses $m_{3} \gg m_{2} \simeq m_{1} \simeq m_{3} \lambda^{4}$. Another choice is the case $\epsilon_{6} \gg \epsilon_{5}$ which corresponds to $m_{3} \gg m_{2} \gg m_{1}$. As a typical case, we take $\epsilon_{6} \simeq \lambda^{2}$ and $\epsilon_{5} \simeq \lambda^{4}$. Then the mixing matrix becomes

$$
V_{9} \simeq\left(\begin{array}{ccc}
-\lambda^{4} & 1 & \lambda^{3} \\
-\lambda^{2} & -\lambda^{3} & 1 \\
1 & \lambda^{4} & \lambda^{2}
\end{array}\right)
$$

with $m_{3} \gg m_{2} \gg m_{1}\left(m_{2} \simeq m_{3} \lambda^{4}, m_{1} \simeq m_{3} \lambda^{8}\right)$. Both cases are consistent with $\Delta m_{32}^{2} \simeq$ $\Delta m_{31}^{2} \simeq \Delta m_{L S N D}^{2}$ and $\Delta m_{21}^{2} \simeq \Delta m_{\odot}^{2}$. Thus, $V_{9}$ could be a natural mass matrix for $m_{3} \gg m_{2} \gg m_{1}$ and $m_{3} \gg m_{2} \simeq m_{1}$.

For $V_{10}$ in eq.(33) we obtain

$$
M_{L L}^{(10)} \simeq\left(\begin{array}{ccc}
m_{1}+m_{2} \epsilon_{3}^{2}+m_{3} \epsilon_{5}^{2} & m_{1} \epsilon_{1}+m_{2} \epsilon_{3} \epsilon_{4}+m_{3} \epsilon_{5} & m_{1} \epsilon_{2}+m_{2} \epsilon_{3}+m_{3} \epsilon_{5} \epsilon_{6} \\
m_{1} \epsilon_{1}+m_{2} \epsilon_{3} \epsilon_{4}+m_{3} \epsilon_{5} & m_{1} \epsilon_{1}^{2}+m_{2} \epsilon_{4}^{2}+m_{3} & m_{1} \epsilon_{1} \epsilon_{2}+m_{2} \epsilon_{4}+m_{3} \epsilon_{6} \\
m_{1} \epsilon_{2}+m_{2} \epsilon_{3}+m_{3} \epsilon_{5} \epsilon_{6} & m_{1} \epsilon_{1} \epsilon_{2}+m_{2} \epsilon_{4}+m_{3} \epsilon_{6} & m_{1} \epsilon_{2}^{2}+m_{2}+m_{3} \epsilon_{6}^{2}
\end{array}\right),
$$


with the naturalness conditions

$$
m_{2} \geq m_{1} \epsilon_{2}^{2}+m_{3} \epsilon_{6}^{2}, \quad m_{1} \geq m_{2} \epsilon_{3}^{2}+m_{3} \epsilon_{5}^{2} .
$$

These relations are held as long as $\epsilon_{6} \geq \epsilon_{5}$. According to the similar analysis given in the previous case $\left(V_{9}\right), V_{10}$ can yield a natural mass matrix.

For $V_{11}$ in eq.(34), we obtain

$$
M_{L L}^{(11)} \simeq\left(\begin{array}{ccc}
m_{1} c^{2}+m_{2} s^{2}+m_{3} \epsilon_{3}^{2} & \left(m_{1}-m_{2}\right) c s+m_{3} \epsilon_{3} \epsilon_{4} & m_{1} \epsilon_{1} c-m_{2} \epsilon_{2} s+m_{3} \epsilon_{3} \\
\left(m_{1}-m_{2}\right) c s+m_{3} \epsilon_{3} \epsilon_{4} & m_{1} s^{2}+m_{2} c^{2}+m_{3} \epsilon_{4}^{2} & m_{1} \epsilon_{1} s+m_{2} \epsilon_{2} c+m_{3} \epsilon_{4} \\
m_{1} \epsilon_{1} c-m_{2} \epsilon_{2} s+m_{3} \epsilon_{3} & m_{1} \epsilon_{1} s+m_{2} \epsilon_{2} c+m_{3} \epsilon_{4} & m_{1} \epsilon_{1}^{2}+m_{2} \epsilon_{2}^{2}+m_{3}
\end{array}\right)
$$

with the naturalness conditions

$$
m_{1} c^{2} \geq m_{2} s^{2}+m_{3} \epsilon_{3}^{2}, \quad m_{2} c^{2} \geq m_{1} s^{2}+m_{3} \epsilon_{3}^{2}
$$

where $c \simeq s \simeq \frac{1}{\sqrt{2}}$. This implies

$$
m_{1} \simeq m_{2}, \quad \frac{m_{2}}{m_{3}} \geq \epsilon_{3}^{2}
$$

i.e. the conditions, under which the mixing matrix $V_{11}$ could lead to a natural mass matrix, but only in the case when $m_{3} \gg m_{2} \simeq m_{1}$.

For $V_{12}$ in eq.(34), we obtain

$$
M_{L L}^{(12)} \simeq\left(\begin{array}{ccc}
m_{1} c^{2}+m_{2} \epsilon_{2}^{2}+m_{3} s^{2} & \left(m_{1}-m_{3}\right) c s+m_{2} \epsilon_{2} \epsilon_{3} & m_{1} \epsilon_{1} c+m_{2} \epsilon_{2}-m_{3} \epsilon_{4} s \\
\left(m_{1}-m_{3}\right) c s+m_{2} \epsilon_{2} \epsilon_{3} & m_{1} s^{2}+m_{2} \epsilon_{3}^{2}+m_{3} c^{2} & m_{1} \epsilon_{1} s+m_{2} \epsilon_{3}+m_{3} \epsilon_{4} c \\
m_{1} \epsilon_{1} c+m_{2} \epsilon_{2}-m_{3} \epsilon_{4} s & m_{1} \epsilon_{1} s+m_{2} \epsilon_{3}+m_{3} \epsilon_{4} c & m_{1} \epsilon_{1}^{2}+m_{2}+m_{3} \epsilon_{4}^{2}
\end{array}\right),
$$

where the naturalness condition is

$$
m_{1} c^{2} \geq m_{2} \epsilon_{2}^{2}+m_{3} s^{2}, \quad m_{2} \geq m_{1} \epsilon_{1}^{2}+m_{3} \epsilon_{4}^{2}
$$

which gives

$$
m_{1} \simeq m_{2}, \quad \frac{m_{2}}{m_{3}} \geq \epsilon_{4}^{2} .
$$

The mixing matrix $V_{12}$ could also give a natural mass matrix for the case of $m_{3} \gg m_{2} \simeq$ $m_{1}$. 
Thus in the case (b) we obtain all together six natural mixing patterns $\left(V_{3,6,9,10,11,12}\right)$ for the hierarchical neutrino masses, and two mixing patterns $\left(V_{5,6}\right)$ for the degenerate masses. The possible solutions for the neutrino masses from the naturalness point of view are summarized in Table2.

\section{Table 2}

In conclusion, we underline that the natural mass matrices with the hierarchical mixing patterns are $V_{2}, V_{3}, V_{6}, V_{9}, V_{10}, V_{11}$ and $V_{12}$. The rest of the patterns, i.e. $V_{1}, V_{4}, V_{5}$, $V_{7}$ and $V_{8}$, lead to the unnatural mass matrices in case of the hierarchical mass matrices. However, if the degenerate mass matrices are allowed as the natural mass matrices, the mixing solutions $V_{1}, V_{5}$ and $V_{6}$ lead to the natural mass matrices at the level of first stage of naturalness. In the next section, we consider the naturalness of the $M_{R R}$ for the selected $V_{1}, V_{2}, V_{3}, V_{5}, V_{6}, V_{9}, V_{10}, V_{11}$ and $V_{12}$ patterns. Here $V_{1}$ is a typical case with the degenerate neutrino masses under condition of naturalness.

\section{Natural see-saw realization}

In this section, we study the natural realization of the neutrino mass matrix in the seesaw mechanism [33]. Without loss of generality we consider $V_{1}$ as a sample of the three degenerate neutrino masses.

Since the mixing patterns $V_{1}, V_{2}, V_{3}, V_{5}$ and $V_{6}$ explain the atmospheric neutrino deficit, the large mixing angle (maximal mixing) is inevitable. Moreover, at least two neutrinos are almost degenerate in these cases. Our aim is to derive the large mixing angle and the degenerate masses from the see-saw mechanism using the hierarchical Dirac mass matrix $M_{L R}$. In general, there are some conditions to reproduce the large mixing by using the hierarchical Dirac mass matrix in the see-saw mechanism. This see-saw enhancement was studied previously by Smirnov and Tanimoto [35]. 
For simplicity, we discuss the two family model. The maximal mixing is generally given by the matrix

$$
M_{L L} \simeq\left(\begin{array}{ll}
a & b \\
b & a
\end{array}\right)
$$

where the eigenvalues are $a \pm b$. Consider two extreme cases. The first one is the hierarchical case $(a \simeq b)$ which is given by the matrix

$$
M_{L L}^{h i e r} \sim\left(\begin{array}{ll}
1 & 1 \\
1 & 1
\end{array}\right)
$$

The second one is the degenerate case $(a \gg b$ or $a \ll b)$ which is generally given by the matrices

$$
M_{L L}^{\operatorname{deg} 1} \sim\left(\begin{array}{ll}
1 & \epsilon \\
\epsilon & 1
\end{array}\right) \quad \text { or } \quad M_{L L}^{\operatorname{deg} 2} \sim\left(\begin{array}{cc}
\epsilon & 1 \\
1 & \epsilon
\end{array}\right)
$$

where $\epsilon \ll 1$. Since the hierarchical case with large angle solution is already excluded from the naturalness in section 2 , we discuss only the degenerate case in this section. The inverse matrix of $M_{L L}^{\operatorname{deg} 1}$ in eq.(75) is

$$
M_{L L}^{-1} \sim\left(\begin{array}{cc}
1 & -\epsilon \\
-\epsilon & 1
\end{array}\right)
$$

If we consider the charged lepton or down quark mass matrix

$$
M_{L R} \sim\left(\begin{array}{cc}
a \lambda^{2} & b \lambda \\
b \lambda & 1
\end{array}\right) \text { with } a, b \simeq \mathcal{O}(1)
$$

as a typical neutrino Dirac mass matrix, we obtain the right-handed Majorana mass matrix

$$
M_{R R}=M_{L R}^{T} M_{L L}^{-1} M_{L R} \sim\left(\begin{array}{cc}
\lambda^{2}\left(b^{2}-2 a b \epsilon \lambda+a^{2} \lambda^{2}\right) & \lambda\left(b-\left(a+b^{2}\right) \epsilon \lambda+a b \lambda^{2}\right) \\
\lambda\left(b-\left(a+b^{2}\right) \epsilon \lambda+a b \lambda^{2}\right) & 1-2 b \epsilon \lambda+b^{2} \lambda^{2}
\end{array}\right) .
$$

Then the large angle solution like eq.(75) is obtained in the case when the factor $\epsilon \lambda$ of all elements in $M_{R R}$ is fine tuned to the leading term. Similar argument is valid also for the case of $M_{L L}^{\operatorname{deg} 2}$.

Since we must require the fine-tuning for the matrix $M_{R R}$, the solutions with the almost degenerate light neutrino masses, which are not excluded by the naturalness of 
the mass matrix, could be excluded by the arguments of naturalness of right-handed Majorana mass matrix in the see-saw realization.

In three family case, the situation is same as in the case of two families. Consider, for example, case(a-1) with the three degenerate masses. Following eq.(42), we obtain

$$
M_{L L}^{-1} \sim\left(\begin{array}{ccc}
1 & -\epsilon & -\epsilon \\
-\epsilon & 1 & -\epsilon \\
-\epsilon & -\epsilon & 1
\end{array}\right)
$$

where $\epsilon_{i}$ 's are taken to be equal to $\epsilon$. If we accept the following natural mass matrices in the quark sector [2]

$$
M_{u} \propto\left(\begin{array}{ccc}
\lambda^{7} & \lambda^{6} & \lambda^{4} \\
\lambda^{6} & \lambda^{4} & \lambda^{4} \\
\lambda^{4} & \lambda^{4} & 1
\end{array}\right), \quad M_{d} \propto\left(\begin{array}{ccc}
\lambda^{4} & \lambda^{3} & \lambda^{5} \\
\lambda^{3} & \lambda^{2} & \lambda^{2} \\
\lambda^{5} & \lambda^{2} & 1
\end{array}\right)
$$

for the Dirac neutrino mass matrix, we obtain

$$
M_{R R} \propto\left(\begin{array}{ccc}
\lambda^{8}\left(1+2 \lambda^{2} \epsilon\right) & \lambda^{8}(1+\epsilon) & \lambda^{4}\left(1+\lambda^{2} \epsilon\right) \\
\lambda^{8}(1+\epsilon) & 2 \lambda^{8}(1+\epsilon) & \lambda^{4}(1+\epsilon) \\
\lambda^{4}\left(1+\lambda^{2} \epsilon\right) & \lambda^{4}(1+\epsilon) & 1+4 \lambda^{4} \epsilon
\end{array}\right) \quad \text { for } \quad M_{L R}=M_{u}
$$

and

$$
M_{R R} \propto\left(\begin{array}{ccc}
\lambda^{6}(1+2 \lambda \epsilon) & \lambda^{5}(1+\epsilon) & 2 \lambda^{3}\left(\lambda^{2}+\epsilon\right) \\
\lambda^{5}(1+\epsilon) & 2 \lambda^{4}(1+\epsilon) & \lambda^{2}(1+\epsilon) \\
2 \lambda^{3}\left(\lambda^{2}+\epsilon\right) & \lambda^{2}(1+\epsilon) & 1+2 \lambda^{2} \epsilon
\end{array}\right) \quad \text { for } \quad M_{L R}=M_{d}
$$

It is found that all the elements in $M_{R R}$ for both cases are fine-tuned to the leading term. Thus, if we take the natural quark mass matrix for the neutrino Dirac mass matrix, which may be justified in GUT's model, we will not be able to obtain the natural neutrino mass matrix for the case with the large angle mixing angle and the degenerate masses.

$V_{2,11,12}$ with the hierarchical solution $m_{3} \gg m_{2} \simeq m_{1}$, and $V_{3,6}$ with $m_{3} \simeq m_{2} \gg m_{1}$ are exactly the same situation as in the above degenerated case(a-1). The hierarchical realization with a large mixing angle needs precisely fine-tuned right-handed Majorana mass structure.

Let us consider another typical mixing pattern $V_{9}$ and $V_{10}$, where the large mixing angle is excluded. Here the mass hierarchy is inverse. For $V_{9}$ the $\mu$-like neutrino is the heaviest one, while the $\tau$-like neutrino is the lightest one. For $V_{10}$ the $\mu$-like neutrino is the heaviest 
one, and the $e$-like neutrino is the lightest one. This inverse mass hierarchy requires the huge mass hierarchy of the right-handed Majorana masses. Assigning the numerical value to $V_{9}, V_{10}$ and neutrino masses with hierarchical structure $m_{3} \gg m_{2} \geq m_{1}$, we found that the huge right-handed Majorana mass hierarchy such as $M_{1} / M_{3} \simeq 10^{4} \sim 10^{6}$ may still be a reasonable hierarchy. For example, in the case $V_{9}$ the left-handed Majorana masses are given by eq.661) and eqs.(63,64), from where we obtain

$$
M_{L L}^{(9)} \propto\left(\begin{array}{ccc}
1 & \lambda^{2} & \lambda^{2} \\
\lambda^{2} & \lambda^{4} & \lambda^{4} \\
\lambda^{2} & \lambda^{4} & \lambda^{4}
\end{array}\right), \quad\left(\begin{array}{ccc}
1 & \lambda^{4} & \lambda^{2} \\
\lambda^{4} & \lambda^{8} & \lambda^{6} \\
\lambda^{2} & \lambda^{6} & \lambda^{4}
\end{array}\right),
$$

for $m_{3} \gg m_{2} \simeq m_{1}$ in eq.(63) and for $m_{3} \gg m_{2} \gg m_{1}$ in eq.(64), respectively. Using this matrix and considering the hierarchical structure as a quark sector for the Dirac neutrino masses $M_{L R}$, we get the right-handed Majorana mass matrices:

$$
\begin{aligned}
M_{R R}^{(9)} & \propto\left(\begin{array}{ccc}
\lambda^{8} & \lambda^{8} & \lambda^{4} \\
\lambda^{8} & \lambda^{8} & \lambda^{4} \\
\lambda^{4} & \lambda^{4} & 1
\end{array}\right) \quad \text { for } \quad M_{L R}=M_{u}, \\
& \propto\left(\begin{array}{ccc}
\lambda^{6} & \lambda^{5} & \lambda^{3} \\
\lambda^{5} & \lambda^{4} & \lambda^{2} \\
\lambda^{3} & \lambda^{2} & 1
\end{array}\right) \quad \text { for } \quad M_{L R}=M_{d}
\end{aligned}
$$

for the case of $m_{3} \gg m_{2} \simeq m_{1}$ and

$$
\begin{aligned}
M_{R R}^{(9)} & \propto\left(\begin{array}{ccc}
\lambda^{8} & \lambda^{6} & \lambda^{4} \\
\lambda^{6} & \lambda^{4} & \lambda^{2} \\
\lambda^{4} & \lambda^{2} & 1
\end{array}\right) \quad \text { for } \quad M_{L R}=M_{u}, \\
& \propto\left(\begin{array}{ccc}
\lambda^{6} & \lambda & \lambda \\
\lambda & 1 & 1 \\
\lambda & 1 & 1
\end{array}\right) \quad \text { for } \quad M_{L R}=M_{d}
\end{aligned}
$$

for the case of $m_{3} \gg m_{2} \gg m_{1}$. For both cases we need the huge right-handed Majorana mass hierarchy like $M_{1} / M_{3} \simeq \mathcal{O}\left(\lambda^{6 \sim 8}\right) \simeq \mathcal{O}\left(10^{4 \sim 6}\right)$. We also obtain similar hierarchy for the case of $V_{10}$. Thus, the natural mass matrix is given by $V_{9}$ and $V_{10}$ on the second stage.

We conclude that the atmospheric neutrino deficit cannot be reconciled with the naturalness. But what happens if the atmospheric neutrino deficit is indeed due to the neutrino oscillation? This means that one should not take the quark mass matrix texture for the Dirac mass matrix of the neutrino. The GUT's relation is not valid at least for 
the neutrino mass matrix. But the flavor symmetry such as $U(1)$ is favored in the framework of the see-saw mechanism. Actually, quasi-degenerate neutrinos have been already obtained from an abelian group symmetry [36]. Zee mass matrix [37] may be suggested as an alternative model without the see-saw mechanism.

\section{Conclusion}

We have studied the neutrino mass hierarchy and mixing in terms of naturalness of the mass matrix. We have succeeded to select a few neutrino mixing patterns, which could lead to the natural neutrino mass matrix. The mixing patterns $V_{1}, V_{4}, V_{5}, V_{7}$ and $V_{8}$ are unnatural for the solution with at least one hierarchical mass relation among three neutrinos. On the contrary, the mixing patterns of $V_{3}, V_{6}, V_{9}, V_{10}, V_{11}$ and $V_{12}$ are the natural ones in the first stage of naturalness. Also in the framework of the three degenerate mass pattern, mixing matrices $V_{1}, V_{5}, V_{6}, V_{11}$ and $V_{12}$ with the large mixing lead to the

natural mass matrices in the first stage of naturalness. However, those patterns except for $V_{9}$ and $V_{10}$, lead to the unnatural right-handed Majorana mass matrix if the natural quark mass matrices are taken for the Dirac neutrino mass matrix in the framework of the see-saw mechanism.

The mixing patterns $V_{9}$ and $V_{10}$ without the large mixing lead to the natural righthanded Majorana mass matrix. In this case there should be a huge mass hierarchy such as $\mathcal{O}\left(10^{4 \sim 6}\right)$ in order to reproduce the inverse mass hierarchy of the light neutrinos.

The naturalness of the neutrino mass matrix will be clarified in the near future due to the data from the numerous neutrino oscillation experiments being carried out.

\section{Acknowledgment}

The authors would like to give special thanks to Prof. C. Jarlskog for her valuable comments and encouragements in writing this paper. We wish to thank Dr. T. Turova for the careful readings of the manuscript and important comments. Also we thank the 
kind hospitality at the Department of Mathematical Physics of LTH, Lund University, where this work was done. One of the authors(M.M) expresses his thanks to the Japan Society of the Promotion of Sciences and the Royal Swedish Academy of Sciences(RSAS) for the exchange program. Also this work is partially supported by a grant from the RSAS. 


\section{References}

[1] M. Kobayashi and T. Maskawa, Prog. Theor. Phys. 49 (1973) 652.

[2] R.D. Peccei and K. Wang, Phys. Rev. D53 (1996) 2712.

[3] GALLEX Collaboration, P. Angelmann et al.,Phys. Lett.327B (1994) 377; 388B (1996) 384;

SAGE Collaboration, J. N. Abdurashitov et al., Phys. Lett. 328B (1994) 234;

Homestake Collaboration, R. Davis et al., Nucl. Phys. B38 (Proc. Suppl.) (1995) 47;

Kamiokande Collaboration, K. S. Hirata et al., Nucl. Phys. B38

(Proc. Suppl.) (1995) 55.

[4] K. S. Hirata et al., Phys. Lett. 205B (1988) 416; 280B (1992) 146;

D. Casper et al., Phys. Rev. Lett. 66 (1991) 2561;

R. Becker-Szendy et al., Phys. Rev. D46 (1992) 3720.

[5] NUSEX Collaboration, M. Aglietta et al., Europhys. Lett. 8 (1989) 611; 15 (1991) 559 ;

SOUDAN2 Collaboration, W. W. M. Allison et al., Phys. Lett. 391B (1997) 491;

M. C. Goodmann, in Neutrino 94, Proceedings of the 16th International Conference on Neutrino Physics and Astrophysics, Eilat, Israel, ed. A. Dar et al., Nucl. Phys. B38 (1995) 337;

Fréjus Collaboration, K. Daum, Z. Phys. C66 (1995) 417;

MACRO Collaboration, S. Ahlen et al., Phys. Lett. 357B (1995) 481.

[6] Y. Fukuda et al., Phys. Lett. 335B (1994) 237.

[7] Y. Totsuka, invited talk at 18th International Symposium on Lepton-Photon Interactions, July 28 - August 1, 1997, Hamburg. 
[8] LSND Collaboration, C. Athanassopoulos et al., Phys. Rev. Lett. 75 (1995) 2650; 77 (1996) 3082; Phys. Rev. C54 (1996) 2685; nucl-ex/9706006 (1997);

J. E. Hill, Phys. Rev. Lett. 75 (1995) 2654.

[9] KARMEN Collaboration, B. Armbruster et al., Nucl. Phys. B38 (Proc. Suppl.) (1995) 235.

[10] K. Winter, Nucl. Phys. B38 (Proc. Suppl.) (1995) 211;

M. Baldo-Ceolin, Nucl. Phys. B35 (Proc. Suppl.) (1994) 450;

L. DiLella, Nucl. Phys. B31 (Proc. Suppl.) (1993) 319.

[11] B. Achkar et al., Nucl. Phys. B434 (1995) 503.

[12] G. S. Vidyakin et al., Pis'ma Zh. Eksp. Thor. Fiz. 59 (1994) 364; JETP Lett. 59 (1994) 390.

[13] R. I. Steinberg, in Proc. of the 5th Int. Workshop on neutrino Telescopes, Venice, Italy, ed. M. Baldo-Ceolin(INFN, Padua, 1993) p.209.

[14] The CHOOZ collaboration, M. Apollonio et al. hep-ex/9711002 (1997).

[15] S. Parke, hep-ph/9304271(Moriond 1993:Part.Phys.:229-236);

L. Camilleri, CERN Preprint, CERN-PPE/94-87(1994);

ICARUS Collaboration, Gran Sasso Lab. Preprint LNGS-94/99-I (1994);

S. Wojcicki, invited talk at 17th Conference on Neutrino Physics and Neutrino Astrophysics, June 13-19, 1996, Helsinki.

[16] Y. Suzuki, invited talk at 17th Conference on Neutrino Physics and Neutrino Astrophysics, June 13-19, 1996, Helsinki.

[17] CDHS Collaboration, F. Dydak et al., Phys. Lett. 134B (1984) 281. 
[18] CCFR Collaboration, I. E. Stockdale et al., Phys. Rev. Lett. 52 (1984) 1384; Z. Phys. C27 (1985) 53.

[19] E531 Collaboration, N. Ushida et al., Phys. Rev. Lett. 57 (1986) 2897.

[20] E776 Collaboration, L. Borodovsky et al., Phys. Rev. Lett. 68 (1992) 274.

[21] CCFR Collaboration, K. S. McFarland et al., Phys. Rev. Lett. 75 (1995) 3993.

[22] H. Minakata, Phys. Lett. 356B (1995) 61; Phys. Rev. D52 (1995) 6630.

[23] S. M. Bilenky, A. Bottino, C. Giunti and C. W. Kim, Phys. Lett. 356B (1995) 273; DFTT 2/96(JHU-TIPAC 96002) (1996).

[24] G. L. Fogli, E. Lisi and G. Scioscia, Phys. Rev. D52 (1995) 5334.

[25] S. T. Petcov and A. Yu. Smirnov, Phys. Lett. 322B (1994) 109;

D. O. Caldwell and R. N. Mohapatra, Phys. Lett. 354B (1995) 371;

G. Raffelt and J. Silk, Phys. Lett, 366B (1996) 429.

[26] M. Tanimoto, Phys. Rev. D53 (1996) 6632.

[27] L. Wolfenstein, Phys. Rev. Lett.51 (1983) 1945.

[28] A. Yu. Smirnov, Invited talk at 28th International Conference on High-energy Physics (ICHEP 96), Warsaw, Poland, 25-31 July, hep-ph/9611465 (1996).

[29] C.Y. Cardall and G.M. Fuller, Phys. Rev. D53 (1996) 4421.

[30] A. Acker and S. Pakvasa, Phys. Lett. 397B (1997) 209.

[31] G. L. Fogli, E. Lisi, D. Montanino and G. Scioscia, Phys. Rev. D56 (1997) 4365.

C. Y. Cardall, G. M. Fuller and D. B. Cline, Phys.Lett. B413 (1997) 246.

[32] G. Giunti, hep-ph/9802201 (1998). 
[33] M. Gell-Mann, P. Ramond and R. Slansky, in Supergravity, Proceedings of the Workshop, Stony Brook, New York, 1979, edited by P. van Nieuwenhuizen and D. Freedmann, North-Holland, Amsterdam, 1979, p.315;

T. Yanagida, in Proceedings of the Workshop on the Unified Theories and Baryon Number in the Universe, Tsukuba, Japan, 1979, edited by O. Sawada and A. Sugamoto, KEK Report No. 79-18, Tsukuba, 1979, p.95.

[34] T. Tomoda, A. Faessler, K. W. Schmid and F. Grümmer, Nucl. Phys. A 452 (1986) 591 ;

J. Engel, P. Vogel, X. Ji and S. Pittel, Phys. Lett. B 225 (1989) 5;

H. V. Klapdor-Kleingrothaus, Nucl. Phys. (Proc.Suppl) B 48 (1996) 216.

[35] A. Yu. Smirnov, Phys. Rev. D48 (1993) 3264;

M. Tanimoto, Phys. Lett. 345B (1995) 477.

[36] P. Binétruy, S. Lavignac, S. Petcov and P. Ramond, Nucl. Phys. B496 (1997) 3.

[37] A. Zee, Phys. Lett. 93B (1980) 389; ibidem 161B (1985) 141;

S. T. Petcov, Phys. Lett. 115B (1982) 401;

S. M. Bilenky and S. T. Petcov, Rev. Mod. Phys. 59 (1987) 671;

L. Wolfenstein, Nucl. Phys. B175 (1980) 93;

A. Y. Smirnov and Z. Tao, Nucl. Phys. B426 (1994) 415;

L. Wolfenstein, CMU-HEP95-05, hep-ph/9506352 (1995);

A. Y. Smirnov and M. Tanimoto, Phys. Rev. D55 (1997) 1665. 


\section{Table 1}

\begin{tabular}{|lc|c|c|}
\hline \multicolumn{2}{|c|}{ case(a) } & Mass relation & Naturalness \\
\hline (1) & $V_{1}$ & $m_{3} \gg m_{2} \gg m_{1}$ & No \\
& & $m_{3} \gg m_{2} \simeq m_{1}$ & No \\
& & $m_{3} \simeq m_{2} \simeq m_{1}$ & Yes \\
\hline$(2)$ & $V_{2}$ & $m_{3} \gg m_{2} \gg m_{1}$ & No \\
& & $m_{3} \gg m_{2} \simeq m_{1}$ & Yes \\
& & $m_{3} \simeq m_{2} \simeq m_{1}$ & Yes(No:2 $\beta$ decay) \\
\hline
\end{tabular}

Table 1: The possible solutions from the naturalness for case(a). The exclusion from other experiments is shown in the parenthesis in the third column.

\section{Table 2}

\begin{tabular}{|cc|c|c|}
\hline \multicolumn{2}{|c|}{ Case(b) } & Mass relation & Naturalness \\
\hline (1) & $V_{3}$ & $m_{3} \simeq m_{2} \gg m_{1}$ & Yes \\
& & $m_{3} \gg m_{2} \simeq m_{1}$ & No \\
& & $m_{3} \gg m_{2} \gg m_{1}$ & No \\
\hline$(1)$ & $V_{4}$ & $m_{3} \gg m_{2} \gg m_{1}$ & No \\
& & $m_{3} \gg m_{2} \simeq m_{1}$ & No \\
\hline$(2)$ & $V_{5}$ & $m_{3} \gg m_{2} \gg m_{1}$ & No \\
& & $m_{3} \gg m_{2} \simeq m_{1}$ & No \\
& & $m_{3} \simeq m_{2} \simeq m_{1}$ & Yes \\
\hline$(2)$ & $V_{6}$ & $m_{3} \simeq m_{2} \gg m_{1}$ & Yes \\
& & $m_{3} \gg m_{2} \simeq m_{1}$ & No \\
& & $m_{3} \gg m_{2} \gg m_{1}$ & No \\
& & $m_{3} \simeq m_{2} \simeq m_{1}$ & Yes \\
\hline$(3)$ & $V_{7}$ & $m_{3} \simeq m_{2} \gg m_{1}$ & No \\
& & $m_{3} \gg m_{2} \simeq m_{1}$ & No \\
\hline$(3)$ & $V_{8}$ & $m_{3} \gg m_{2} \gg m_{1}$ & No \\
& & $m_{3} \gg m_{2} \simeq m_{1}$ & No \\
\hline$(3)$ & $V_{9}$ & $m_{3} \gg m_{2} \gg m_{1}$ & Yes \\
& & $m_{3} \gg m_{2} \simeq m_{1}$ & Yes \\
\hline$(3)$ & $V_{10}$ & $m_{3} \gg m_{2} \gg m_{1}$ & Yes \\
& & $m_{3} \gg m_{2} \simeq m_{1}$ & Yes \\
\hline$(3)$ & $V_{11}$ & $m_{3} \gg m_{2} \gg m_{1}$ & No \\
& & $m_{3} \gg m_{2} \simeq m_{1}$ & Yes \\
\hline$(3)$ & $V_{12}$ & $m_{3} \gg m_{2} \gg m_{1}$ & No \\
& & $m_{3} \gg m_{2} \simeq m_{1}$ & Yes \\
\hline
\end{tabular}

Table 2: The possible solutions from the naturalness for case(b). 